\title{
Efficiency and Blood Loss of Various Spacer and Intramedullary Dowel Constructs in Two-Stage Treatment for Total Knee Arthroplasty Infection
}

\author{
Hanna House BS ${ }^{1}$, Mary Ziemba-Davis BA ${ }^{2}$, R. Michael Meneghini MD³ \\ ${ }^{1}$ Indiana University School of Medicine \\ ${ }^{2}$ IU Health Saxony Orthopedics \\ ${ }^{3}$ Indiana University School of Medicine, Department of Orthopaedic Surgery
}

\section{Background and Hypothesis:}

Treatment for infected total knee arthroplasty (TKA) employs antibiotic-eluding articulating or static spacers, with or without intramedullary (IM) dowels between implant resection and reimplantation. While it is unknown which spacer type is more efficient intra-operatively, IM dowels require additional time for fabrication. Surgical efficiency is critical to minimizing anesthesia time and blood loss, especially in complex surgeries with compromised hosts. We quantified operative time and postoperative intra-articular blood loss based on spacer type and the use of IM dowels.

\section{Project Methods:}

103 consecutive infected TKAs treated from 2010-2019 were retrospectively reviewed. Outcome variables included operative time and intra-articular drain rate. Covariates included sex; age, BMI; ASA-PS classification; surgeon; McPherson infection classification; tourniquet time; tranexamic acid (TXA) use; intrathecal anesthesia, length of stay, and blood transfusion. Multivariate analyses were used.

\section{Results:}

The sample was $52 \%$ female with average age of $66 \pm 9$ years and average BMI of $36 \pm 9 \mathrm{~kg} / \mathrm{m}^{2}$. Articulating spacers without dowels (ASwoD), articulating spacers with dowels (ASwD), and static spacers with dowels were used in $57.3 \%, 21.4 \%$, and $21.4 \%$ of knees, respectively. Longer mean operating time was observed when static spacers with dowels were used at resection (162 vs.130 ASwoD/140 ASwD minutes; $p=0.001$ ) and reimplantation (187 vs. 149 ASwoD/148 ASwD minutes; $p=0.017$ ). At reimplantation, drain rate was highest when articulating spacers with dowels were used (37 vs. $20 / 26 \mathrm{~mL} / \mathrm{hr}$ ), but not when TXA was used $(p=0.002)$.

\section{Conclusion and Potential Impact:}

Articulating and static spacers provide equivalent infection eradication, and the necessity of IM dowels has not been thoroughly studied. In light of this equivalency, it is important to understand other costs associated with spacer types and IM dowels. Our observations that spacer/dowel constructs affect time under anesthesia and blood loss may contribute to the efficiency and safety of the two-stage treatment protocol. 